\title{
Rate and predictive parameters of novel Coronavirus 2019 (Sars-CoV-2) infections in a German General Practice
}

\author{
Moritz Paar $^{1,2}$ (1) $\cdot$ Christoph Strumann ${ }^{3} \cdot$ Heinz Giesen ${ }^{1,2}$
}

Received: 5 November 2020 / Accepted: 9 February 2021 / Published online: 16 February 2021

(c) Royal Academy of Medicine in Ireland 2021

\begin{abstract}
Key Points In our clinical cross-sectional study, we identified 107 of 347 patients who were tested positive for antibodies of novel Coronavirus 2019 (SARS-CoV-2). Main symptoms were exhaustion and cough, exposition to other COVID-19-patients appeared frequently.

Background There is urgent need for information on predictive parameters on immunity and infectivity in Coronavirus disease-2019 (COVID-19) pandemic. Our aim was to investigate distribution of novel Coronavirus 2019 (SARS-CoV-2) infections in a German General Practice and to learn about possible predictive parameters regarding infection and pathways of transmission.

Methods In our cross-sectional study, we tested 347 patients of our General Practice using 2019-nCoV-2-IgG/IgM antibody test [2019-nCoV2 IgG/IgM Rapid Test Cassette (Ref.: INCP-402/INCP-402B; ACRO, BIOTECH, INC.)]. We asked for 13 specific symptoms and 4 questions to investigate patients' surroundings.

Results A total of 107 of 347 patients were tested positive for antibodies (Immunoglobulin M-positive and/or Immunoglobulin G-positive). In antibody-positive group, body aches and rhinorrhea were seen more often and there were significantly less asymptomatic patients. Stay in area of risk was significantly more frequent in antibody-positive group as well as contact to infected persons. Distribution of other symptoms was not significantly different between both groups. Most adults or children with SARS-CoV-2 infection presented with mild flu-like symptoms.

Conclusion A total of $30 \%$ of patients had antibodies. It was not possible to identify one solid predictive symptom. Serological testing may be helpful for the diagnosis of suspected patients with negative RT-PCR results and for the identification of asymptomatic infections.
\end{abstract}

Keywords Antibodies $\cdot$ COVID-19 $\cdot$ Exhaustion $\cdot$ Pandemic $\cdot$ SARS-CoV-2

\section{Background}

2019 novel coronavirus (2019-nCoV; SARS-CoV-2) is a great challenge as it is an ongoing pandemic disease since late 2019 and emerged from China towards the whole world.

Moritz Paar

paarm@uni-muenster.de

Christoph Strumann

c.strumann@uni-luebeck.de

Heinz Giesen

giesenh@uni-muenster.de

1 University Hospital Münster, Münster, Germany

2 General Practice Dr. Dr. Giesen, Ahaus-Wüllen, Germany

3 University Hospital Schleswig-Holstein, Campus Lübeck, Lübeck, Germany
The SARS-CoV-2-virus can cause an acute infection of the respiratory tract $[1,2]$ as well as mild upper respiratory tract symptoms, which suggest the potential for pre- or oligosymptomatic transmission [3-5]. As the world faces a great pandemic, there is urgent need for information on predictive parameters on immunity and infectivity. The identification of predictive parameters has implications for the containment of COVID-19. Current practice for diagnosis of SARSCoV-2 infection relies on PCR testing of nasopharyngeal or respiratory specimens $[6,7]$ in a symptomatic patient at high epidemiologic risk. The virus primarily spreads through the respiratory tract, by droplets, respiratory secretions, and direct contact [8]. Furthermore, presence of SARS-CoV-2 was found in blood and fecal swabs, suggesting the possibility of multiple route transmission $[9,10]$. Incubation period is $1-14$ days, mostly 3-7 days, based on current 
epidemiological investigation. COVID-19 is contagious during the latency period [9]. Patients present certainly similar symptoms, such as fever, exhaustion, and cough as far as we know $[10,11]$. Some patients rapidly develop acute respiratory distress syndrome, respiratory failure, multiple organ failure, even deaths [11-13]. Germany faced a first wave of infections in March and April of 2020. So far (8th of February 2021), 2,288,545 persons were infected and 61,675 died after (COVID-19) [14]. There is urgent need to identify crucial factors of distribution and predictive parameters of ways of infections to contain the pandemic, since real-life sensitivity of PCR testing of nasopharyngeal or respiratory specimens is poor [15]. In our study, we investigated the distribution of infection rate among patients of a general practice. The aim was to identify whether certain symptoms are associated to greater likelihood of COVID-19 and if it is possible to outline risk factors and predictive parameters for infection.

Here, we report an analysis from a German General Practice in the Western part of North-Rhine Westphalia (AhausWuellen) to learn about possible predictive parameters regarding infection and pathways of transmission.

\section{Methods}

We analyzed distribution of COVID-19 using antibody tests and defined time of study duration for 3 months. Patients did contact our general practice voluntarily because they assumed to have overcome COVID-19 or had been in contact to infected persons. A total of 347 people (322 adults and 25 children [defined as persons younger than 20 years]) participated for implementation of a 2019-nCoV-2-IgG/IgM antibody test. We used 2019-nCoV-2 IgG/IgM Rapid Test Cassette (Ref.: INCP-402/INCP-402B; ACRO, BIOTECH, INC.) [sensitivity $96.9 \%$, specificity $96.3 \%$ ]. Our study had three possible inclusion criteria:

(A) symptoms of acute respiratory infection that had ended at least 2 weeks before OR.

(B) contact to a COVID-19 patient at least 2 weeks before OR.

(C) criteria (A) and (B) were matched.

Each patient who matched criteria (A), (B), or (C) signed official consent of participation as well as private policy and data processing agreement. Every patient had to complete a questionnaire for retrospective processing of symptoms that was developed by the authors according to official questionnaire of German Association of General Medicine [16]. The questionnaire is shown as Fig. 1.

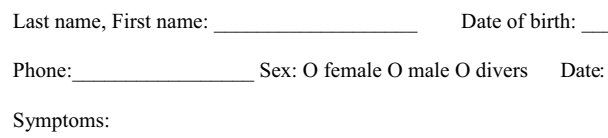

\begin{tabular}{|c|c|c|c|c|}
\hline Symptom & Yes & No & Time & \\
\hline sudden start? & $\mathrm{O}$ & $\mathrm{O}$ & from & to \\
\hline $\begin{array}{l}\text { shortness of breath at } \\
\text { rest? }\end{array}$ & $\mathrm{O}$ & $\mathrm{O}$ & from & to \\
\hline $\begin{array}{l}\text { shortness of breath at } \\
\text { stress? }\end{array}$ & $\mathrm{O}$ & $\mathrm{O}$ & from & to \\
\hline fever? & $\mathrm{O}$ & $\mathrm{O}$ & from & to \\
\hline exhaustion? & $\mathrm{O}$ & $\mathrm{O}$ & from & to \\
\hline cough? & $\mathrm{O}$ & $\mathrm{O}$ & from & to \\
\hline body aches? & $\mathrm{O}$ & $\mathrm{O}$ & from & to \\
\hline sore throat? & $\mathrm{O}$ & $\mathrm{O}$ & from & to \\
\hline rhinorrhea? & $\mathrm{O}$ & $\mathrm{O}$ & from & to \\
\hline \begin{tabular}{|l|} 
headache? \\
\end{tabular} & $\mathrm{O}$ & $\mathrm{O}$ & from & to \\
\hline diarrhea? & $\mathrm{O}$ & $\mathrm{O}$ & from & to \\
\hline swallowing disorder? & $\mathrm{O}$ & $\mathrm{O}$ & from & to \\
\hline skin rush? & $\mathrm{O}$ & $\mathrm{O}$ & from & to \\
\hline
\end{tabular}

Further questions:

\begin{tabular}{|l|l|}
\hline travel history in past 2 weeks? & O yes, where? \\
\hline stay in area of risk for COVID-19 in past 2 & O yes, where? \\
weeks (according to criteria of Robert- & O no \\
Koch-Institute from March 24th, 2020)? & \\
\hline direct contact to COVID-19-patient in past 2 & O yes, where, when? \\
weeks? & O no \\
\hline direct contact to supposed COVID-19- & O yes, where, when? \\
patient in past 2 weeks? & O no \\
\hline
\end{tabular}

Fig. 1 Questionnaire

Patients' index finger or middle finger was disinfected and punctured with a sterile lancet. Fingerstick whole blood specimen of $20 \mu \mathrm{L}$ was collected and added to the specimen well of the test cassette. Two drops of buffer solution (approximately $80 \mu \mathrm{L}$ ) were added to the specimen well. Results were noted after exactly $10 \mathrm{~min}$.

Possible test results were antibody negative (neither Immunoglobulin G-positive nor Immunoglobulin M-positive), Immunoglobulin G-positive (IgG-pos), Immunoglobulin M-positive (IgM-pos) or Immunoglobulin G- and Immunoglobulin M-positive (IgG- and IgM-pos). We statistically 
analyzed correlation between positive antibody test and symptoms as well as combination of symptoms. Statistical analyses were performed with STATA 15 (StataCorp LLC, College Station, TX, USA).

\section{Results}

We included 347 patients in our analysis that were tested with a 2019-nCoV-2-IgG/IgM antibody test in our general practice (see Table 1 ).

The mean age of the participants was 45.2 years and 191 (55\%) were females. Among the participants, 25 were children with a mean age of 11.6 years (14 males and 11 females). In total, 107 (30.8\%) patients were tested positive for antibodies (IgG-pos, IgM-pos, or IgG- and IgMpos). Out of these, their mean age was 50.2 years and 51 (48\%) were female patients.

The number of patients tested IgM-pos was 31 (9.9\%) with mean age of 50.3 years, 15 female (48\%) patients. Sixty-five (18.7\%) patients were tested only IgG-pos with mean age of 41.8 years, 30 females (46\%). Eleven (3.2\%) patients were tested IgG-pos and IgM-pos at the same time with a mean age of 46.5 years, 6 females $(54.5 \%)$. Further, we observed 240 patients $(69.2 \%)$ with a negative test result. Their mean age was 45.7 years, 140 females (58\%).

Eighteen out of 25 children were tested antibodynegative ( 9 males, 9 females; mean 10.6 years). Seven children were tested IgG-pos ( 5 males, 2 females; mean 14.1 years). There were no children tested IgM-pos or IgGand IgM-pos.
Only one out of 347 patients had to be treated in Intensive Care Unit. Six patients had to be treated in hospital. Most patients with SARS-CoV-2 infection presented with mild flu-like symptoms and had good prognosis. Distribution of these symptoms is shown in Table 2 .

In antibody-positive group, there were significantly less asymptomatic patients $(9.3 \%)$ than in antibody-negative group (22.9\%) ( $p$-value 0.004$)$. Symptom of body aches appeared in $50.5 \%$ of antibody-positive tested persons, compared with $37.9 \%$ of antibody-negative tested ( $p$-value $0.029)$. Rhinorrhea was seen in $52.3 \%$ of antibody-positive patients and in $40.8 \%$ of antibody-negative patients ( $p$-value $0.046)$. Stay in area of risk was significantly more frequent in antibody-positive group (18.7\%) than in antibodynegative group (10.8\%) ( $p$-value 0.046$)$. Contact to infected person appeared more often in antibody-positive group $(52.3 \%)$ compared with antibody-negative group (31.3\%) ( $p$ value $<0.001)$. We found exhaustion or cough more often in antibody-positive group (81.3\%) than in antibody-negative group (70.0\%) ( $p$-value 0.028 ). Exhaustion or cough or body aches was seen in $83.2 \%$ of antibody-positive patients and in $70.8 \%$ of antibody-negative patients ( $p$-value 0.015 ). Cough or body aches were identified in $76.6 \%$ of antibody-positive patients and in $65.4 \%$ of antibody negative patients ( $p$-value 0.037 ). Distribution of other symptoms was not significantly different between both groups and can be found in Table 3 .

Twenty-two patients out of our study had received PCR testing independently to our study because of infection symptoms a couple of weeks before we tested for antibodies (patients gave consent for publication of results). Nine patients had a negative PCR test as well as negative antibody test more than 2 weeks
Table 1 Distribution of gender and age

\begin{tabular}{llllll}
\hline & Total $(n=347)$ & $\begin{array}{l}\text { Antibody-neg- } \\
\text { ative }(n=240)\end{array}$ & IgG-pos $(n=65)$ & IgM-pos $(n=31)$ & $\begin{array}{l}\text { IgG- and } \\
\text { IgM-pos } \\
(n=11)\end{array}$ \\
\hline All & $n(\%)$ & $n(\%)$ & $n(\%)$ & $n(\%)$ & $n(\%)$ \\
Female & $191(55.0)$ & $140(58.3)$ & $30(46.2)$ & $15(48.4)$ & $6(54.5)$ \\
Male & $156(45.0)$ & $100(41.7)$ & $35(53.8)$ & $16(51.6)$ & $5(45.5)$ \\
Mean age & 45.2 & 45.7 & 41.0 & 50.3 & 46.4 \\
Age (min-max) & $4-79$ & $4-79$ & $10-72$ & $25-79$ & $24-59$ \\
Children (age $<20)$ & & & & & \\
Female & $11(44.0)$ & $9(50.0)$ & $2(28.6)$ & $0(0.0)$ & $0(0.0)$ \\
Male & $14(56.0)$ & $9(50.0)$ & $5(71.4)$ & $0(0.0)$ & $0(0.0)$ \\
Mean age & 11.6 & 10.6 & 14.1 & - & - \\
Age (min-max) & $4-19$ & $4-18$ & $10-19$ & - & - \\
\hline
\end{tabular}

IgG-pos Immunoglobulin G-positive, IgM-pos Immunoglobulin M-positive, IgG- \& IgM-pos Immunoglobulin G- and Immunoglobulin M-positive

Data are in $\%$ unless otherwise indicated

${ }^{\mathrm{a}}$ Odds ratio (relative risk) of having a positive antibody test result

${ }^{\mathrm{b}}$ Pearson $x^{2}$ test $p$-value

${ }^{\mathrm{c}} t$ test $p$-value 
Table 2 Distribution of symptoms

\begin{tabular}{|c|c|c|c|c|}
\hline Symptom & Test negative $(n=240)$ & IgG-pos $(n=65)$ & $\operatorname{IgM}-\operatorname{pos}(n=31)$ & $\begin{array}{l}\text { IgG- \& } \\
\text { IgM-pos } \\
(n=11)\end{array}$ \\
\hline & $n(\%)$ & $n(\%)$ & $n(\%)$ & $n(\%)$ \\
\hline Asymptomatic & $57(23.85)$ & $9(13.85)$ & $3(9.68)$ & $1(9.09)$ \\
\hline Sudden start & $94(39.17)$ & $33(50.77)$ & $16(51.61)$ & $4(36.36)$ \\
\hline Dyspnea at rest & $25(10.42)$ & $11(16.92)$ & $4(12.90)$ & $3(27.27)$ \\
\hline Dyspnea at stress & $57(23.75)$ & $19(29.23)$ & $7(22.58)$ & $4(36.36)$ \\
\hline Fever & $48(20.00)$ & $17(26.15)$ & $4(12.90)$ & $2(18.18)$ \\
\hline Exhaustion & $142(59.17)$ & $44(67.69)$ & $21(67.74)$ & $9(81.82)$ \\
\hline Cough & $139(57.92)$ & $41(63.08)$ & $20(64.52)$ & $4(36.36)$ \\
\hline Body ache & $91(37.92)$ & $35(53.85)$ & $13(41.94)$ & $6(54.55)$ \\
\hline Sore throat & $123(51.25)$ & $33(50.77)$ & $18(58.06)$ & $5(45.45)$ \\
\hline Rhinorrhea & $98(40.83)$ & $35(53.85)$ & $15(48.39)$ & $6(54.55)$ \\
\hline Headache & $111(46.25)$ & $34(52.31)$ & $15(48.39)$ & $7(63.64)$ \\
\hline Diarrhea & $50(20.83)$ & $18(27.69)$ & $4(12.90)$ & $6(54.55)$ \\
\hline Swallowing disorder & $60(25.00)$ & $16(24.62)$ & $9(29.03)$ & $2(18.18)$ \\
\hline Skin rush & $10(4.17)$ & $4(6.15)$ & $4(12.90)$ & $2(18.18)$ \\
\hline Travel history in past 2 weeks & $44(18.33)$ & $20(30.77)$ & $3(9.68)$ & $3(27.27)$ \\
\hline Stay in area of risk for COVID-19 in past 2 weeks & $26(10.83)$ & $16(24.62)$ & $1(3.23)$ & $3(27.27)$ \\
\hline Direct contact to COVID-19-patient in past 2 weeks & $75(31.25)$ & $41(63.08)$ & $9(29.03)$ & $6(54.55)$ \\
\hline Direct contact to supposed COVID-19-patient in past 2 weeks & $42(17.50)$ & $18(27.69)$ & $6(19.35)$ & $4(36.36)$ \\
\hline
\end{tabular}

No. (\%), IgG-pos Immunoglobulin G-positive, IgM-pos Immunoglobulin M-positive, IgG- \& IgM-pos Immunoglobulin G- and Immunoglobulin M-positive

after PCR (40.9\%). For 10 patients with a negative PCR test, we were able to find antibodies more than 2 weeks later $(45.5 \%)$, in 7 of these patients' result was IgG-pos, in 3 cases we found IgM. There were 3 patients $(13.6 \%)$ with positive PCR test and IgG-pos more than 2 weeks later.

\section{Discussion}

\section{Limitations}

We present a selected study group as patients did contact our general practice voluntarily because they assumed to have overcome COVID-19 or had been in contact to infected persons. Of course, results might be affected by this as well as by the fact that people had to pay for the antibody test. Further, there is a certain demographic and geographic pre-selection as all patients live in a rural area. Health-conscious behavior might be different from rural to metropolitan area. Therefore, results cannot basically be transferred on the whole country. In addition, number of children tested was small, as discussed later.

\section{Symptoms}

By the time we run through our study, there was less experience worldwide regarding implementation of SARS-CoV-2 antibody tests. We were able to identify exhaustion and cough as main symptoms in the group of IgG-pos patients. Most of IgG-pos patients had been exposed to other COVID19 patients or stayed in an area of risk. It was not possible to differentiate significantly between antibody-positive and antibody-negative persons using a certain single symptom. On the other hand, we found that antibody-negative tested persons were more frequently asymptomatic in comparison with antibody-positive tested. Symptoms were similar compared with big analyses of Huang and Cervia [17, 18]. As far as we know, underlying health conditions as pulmonary disease, diabetes, and old age are associated with severe course of COVID-19 [19]. SARS-CoV-2 responses are known to be stronger and broader in individuals with more severe COVID-19, but these show weaker antibody responses to prior infections. Among hospitalized patients, males produce stronger SARS-CoV-2 antibody responses than females [20].

\section{Children and adults}

Human-to-human transmission of SARS-CoV-2 mainly occurs between family members, including relatives and friends in intimate contact with patients or incubation carriers [21]. We were not able to identify a significant rate of infections in certain families. Rate of infections in different family surroundings was between 25 and $100 \%$. We were 
Table 3 Significance of symptoms

\begin{tabular}{|c|c|c|c|c|c|}
\hline & Total $(n=347)$ & $\begin{array}{l}\text { Antibody-nega- } \\
\text { tive }(n=240)\end{array}$ & $\begin{array}{l}\text { Antibody-posi- } \\
\text { tive }(n=107)\end{array}$ & Odds ratio $^{a}$ & $p$-value $^{\mathrm{b}}$ \\
\hline & $n(\%)$ & $n(\%)$ & $n(\%)$ & & \\
\hline \multicolumn{6}{|l|}{ Sociodemography } \\
\hline Female & $191(55.0)$ & $140(58.3)$ & $51(47.7)$ & 0.65 & 0.065 \\
\hline Male & $156(45.0)$ & $100(41.7)$ & $56(52.3)$ & 1.54 & 0.065 \\
\hline Mean age & 45.2 & 45.7 & 44.2 & 0.99 & .43 \\
\hline \multicolumn{6}{|l|}{ Symptoms } \\
\hline Asymptomatic & $65(18.7)$ & $55(22.9)$ & $10(9.3)$ & 0.35 & .004 \\
\hline Sudden start & $147(42.4)$ & $94(39.2)$ & $53(49.5)$ & 1.52 & .071 \\
\hline Dyspnea at rest & $43(12.4)$ & $25(10.4)$ & $18(16.8)$ & 1.74 & .094 \\
\hline Dyspnea at stress & $87(25.1)$ & $57(23.8)$ & $30(28.0)$ & 1.25 & .39 \\
\hline Fever & $71(20.5)$ & $48(20.0)$ & $23(21.5)$ & 1.10 & .75 \\
\hline Exhaustion & $216(62.2)$ & $142(59.2)$ & $74(69.2)$ & 1.55 & .076 \\
\hline Cough & $204(58.8)$ & $139(57.9)$ & $65(60.7)$ & 1.13 & .62 \\
\hline Body ache & $145(41.8)$ & $91(37.9)$ & $54(50.5)$ & 1.67 & .029 \\
\hline Sore throat & $179(51.6)$ & $123(51.3)$ & $56(52.3)$ & 1.04 & .85 \\
\hline Rhinorrhea & $154(44.4)$ & $98(40.8)$ & $56(52.3)$ & 1.59 & .046 \\
\hline Headache & $167(48.1)$ & $111(46.3)$ & $56(52.3)$ & 1.28 & .30 \\
\hline Diarrhea & $78(22.5)$ & $50(20.8)$ & $28(26.2)$ & 1.35 & .27 \\
\hline Swallowing disorder & $87(25.1)$ & $60(25.0)$ & $27(25.2)$ & 1.01 & .96 \\
\hline Skin rush & $20(5.8)$ & $10(4.2)$ & $10(9.3)$ & 2.37 & .056 \\
\hline \multicolumn{6}{|l|}{ Combination of symptoms } \\
\hline Exhaustion and cough & $165(47.6)$ & $113(47.1)$ & $52(48.6)$ & 1.06 & 0.79 \\
\hline Exhaustion OR cough & $255(73.5)$ & $168(70.0)$ & $87(81.3)$ & 1.86 & 0.028 \\
\hline Exhaustion and sore throat & $143(41.2)$ & $97(40.4)$ & $46(43.0)$ & 1.11 & 0.65 \\
\hline Exhaustion OR sore throat & $252(72.6)$ & $168(70.0)$ & $84(78.5)$ & 1.57 & 0.10 \\
\hline Exhaustion and cough and body aches & $100(28.8)$ & $67(27.9)$ & $33(30.8)$ & 1.15 & 0.58 \\
\hline Exhaustion OR cough OR Body aches & $259(74.6)$ & $170(70.8)$ & $89(83.2)$ & 2.04 & 0.015 \\
\hline Cough and body aches & $110(31.7)$ & $73(30.4)$ & $37(34.6)$ & 1.21 & 0.44 \\
\hline Cough OR body aches & $239(68.9)$ & $157(65.4)$ & $82(76.6)$ & 1.73 & 0.037 \\
\hline Cough and sore throat & $147(42.4)$ & $102(42.5)$ & $45(42.1)$ & 0.98 & 0.94 \\
\hline Cough OR sore throat & $236(68.0)$ & $160(66.7)$ & $76(71.0)$ & 1.23 & 0.42 \\
\hline Exhaustion and headache & $140(40.3)$ & $90(37.5)$ & $50(46.7)$ & 1.46 & 0.11 \\
\hline Exhaustion OR headache & $243(70.0)$ & $163(67.9)$ & $80(74.8)$ & 1.40 & 0.20 \\
\hline \multicolumn{6}{|l|}{ Behavior prior to test } \\
\hline Travel history in past 2 weeks & $70(20.2)$ & $44(18.3)$ & $26(24.3)$ & 1.43 & .20 \\
\hline Stay in area of risk for COVID-19 in past 2 weeks & $46(13.3)$ & $26(10.8)$ & $20(18.7)$ & 1.89 & .046 \\
\hline Direct contact to COVID-19-patient in past 2 weeks & $131(37.8)$ & $75(31.3)$ & $56(52.3)$ & 2.42 & $P<.001$ \\
\hline Direct contact to supposed COVID-19-patient in past 2 weeks & $70(20.2)$ & $42(17.5)$ & $28(26.2)$ & 1.67 & .0631 \\
\hline
\end{tabular}

Data are in $\%$ unless otherwise indicated

${ }^{a}$ Odds ratio (relative risk) of having a positive antibody test result

${ }^{\mathrm{b}}$ Pearson $x^{2}$ test $p$-value

${ }^{\mathrm{c}} t$ test $p$-value

limited by the fact that we only saw single family members in most cases. Many persons did not want their relatives to be tested as well. Most patients had mild symptoms, especially younger people, as already seen in other studies [22]. Children are reported to have a better prognosis and pediatric COVID-19 infection is relatively mild when compared with 
adults [23]. In our study, 7 children were tested IgG-pos with mild infection. We were not able to include a big number of children in our analysis due to different reasons: most children in Germany are treated in pediatric practice until age of 16 to 18 years, so we do not see many children in general practice. Additionally, many parents preferred to isolate their children at home during first wave of infection to avoid any risk of infection that might occur in a practice during pandemic. Finally, most parents probably did not suspect an infection of their children due to very mild symptoms and therefore did not see a necessity for testing [23, 24].

\section{Cross-reactivity}

As already known, there are common human coronavirus strains and other common respiratory viruses [17]. Binding of an antibody on two different antigens that show similar binding sites might lead to false-positive results. Cross-reactivity of IgM-antibodies might be possible against endemic (Beta-) Coronaviruses. This might cause potential false-positive results regarding IgM-pos patients [25]. Other molecules, including interferon, rheumatoid factor, non-specific IgM, and so on might as well cause false-positive results. There is probably a cross-reactivity between the N protein of SARS-CoV-2 and antibodies against other human coronaviruses [26]. For these reasons, we are skeptical regarding results of IgM-pos patients since possible cross-reactivity might occur. We conclude that detection sensibility is higher in IgG-IgM combined antibody tests than in individual IgG or IgM antibody tests [27].

The median seroconversion time for IgM and then IgG is day 12 and day 14 as shown by Cervia [18] and Zhao [28]. Thus, possibly, some patients might have been detected before seroconversion of $\mathrm{IgG}$ had occurred. If antibody testing is done during the early stage of the infection, they may produce false-negative results [27]. So, we are confident to state that antibody testing should not be used to early after beginning of symptoms or contact to a COVID-19 patient.

\section{Discussion about polymerase chain reaction vs. antibody test}

In clinical practice of outpatient care, there are certain challenges regarding quality and availability of polymerase chain reaction (PCR) from nasal and throat swab which need to be discussed. It is known that nucleic acid-based methods are sensitive but prone to false-positive results [29]. Quality of PCR depends on different aspects and we address specific questions about this method: at what time is tested after beginning of symptoms or after contact to a COVID19-person? How is the quality of the sample regarding depth of swap and amount of material? How long does it take to transport a sample from a Practice in a rural area to a central laboratory? Do criteria of Robert-Koch-Institute allow testing at the expense of health insurance/does patient have to pay for testing? How is quality of laboratory procedure?

Especially in rural regions, tests were not available nationwide at the beginning of pandemic in Germany. Further, type of indication position for testing due to recommendation of the Robert-Koch-Institute (RKI) was adjusted multiple times [7]. Likewise, period between beginning of symptoms and testing charges quality of results. Finally, quality of testing considering operation process of testing shows big variety. Thus, PCR testing likely underestimates the true prevalence of infection, creating the need for serologic methods to detect infections [30, 31]. Findings of our daily practice routine support this assumption as described above: 10 out of 22 patients with a negative PCR were tested positive for antibodies more than 2 weeks later $(45,45 \%)$; in 7 of these patients, result was IgG-pos; in 3 cases, we found IgM. These findings support importance of quarantine for symptomatic patients as well as for patients who were in contact to infected persons, even if PCR test is negative. As median seroconversion time for $\operatorname{IgM}$ and then $\operatorname{IgG}$ is about 12-14 days [18, 28], antibody testing after time of quarantine might be helpful to identify cases of COVID-19. Nevertheless, it is important to test via PCR when infection is suspected. The antibody-based method has slightly lower sensitivity but higher accuracy than PCR [26, 27, 29]. Compared with PCR, antibody testing saves time and does not require equipment, it is simple to perform, and only requires minimal training. Another potential application of this test is screening asymptomatic SARS-CoV-2 carriers [27, 29]. Therefore, it is suggested to combine the two methods to improve the detection accuracy of COVID-19. We conclude that serological testing may be helpful for the diagnosis of suspected patients with negative RT-PCR results and for the identification of asymptomatic infections [26-29].

Much more progress is necessary in the characterization of the novel coronavirus and the development of therapies and vaccines against the virus. Even if PCR testing, which is usually used at point of suspected infection, is negative, one cannot exclude infection [26-29]. We conclude that even patients with negative PCR testing should be advised to take care of social distancing, wearing face mask, and measures of hygienics. At this point of pandemic, there is no alternative of action.

Author contribution Each author has participated sufficiently in the work to take public responsibility for the content.

\section{Declarations}

Ethical approval All procedures performed in studies involving human participants were in accordance with the ethical standards of the institutional and/or national research committee and with the 1964 Helsinki declaration and its later amendments or comparable ethical standards. 
Consent to participate and consent for publication Patients voluntary contacted our general practice, received reconnaissance, signed consent of participation and consent for publication.

Informed consent Informed consent was obtained from all individual participants included in the study.

Conflict of interest The authors declare that patients voluntary contacted our general practice, received reconnaissance, signed consent of participation, and paid 60 Euro for implementation and evaluation of antibody testing as well as explanation of results. There are no further conflicts of interest to declare.

\section{References}

1. Zhu N, Zhang D, Wang W et al (2020) A novel coronavirus from patients with pneumonia in China, 2019. N Engl J Med 382:727-733

2. Gorbalenya A, Baker S, Baricet R et al (2020) Severe acute respiratory syndrome-related coronavirus: the species and its viruses - a statement of the Coronavirus Study Group. Preprint at https://www.biorxiv.org/content/https://doi. org/10.1101/2020.02.07.937862v1

3. Rothe C, Schunk M, Sothmann P et al (2020) Transmission of 2019-nCoV infection from an asymptomatic contact in Germany. N Engl J Med 382:970-971

4. Holshue M, DeBolt C, Lindquist S et al (2020) First case of 2019 novel coronavirus in the United States. N Engl J Med 382:929-936

5. Hoehl S, Rabenau H, Berger A et al (2020) Evidence of SARS$\mathrm{CoV}-2$ infection in returning travelers from Wuhan. China N Engl J Med 382:1278-1280

6. Zhen W, Manji R, Smith E et al (2020) Comparison of four molecular in vitro diagnostic assays for the detection of SARS-CoV-2 in nasopharyngeal specimens. J Clin Microbiol 58(8):e00743-e820. https://doi.org/10.1128/JCM.00743-20

7. Homepage of Robert-Koch-Institute, Germany (2021) Criteria of testing for SARS-CoV-2. Daily update. https://www.rki.de/ DE/Content/InfAZ/N/Neuartiges_Coronavirus/Massnahmen_ Verdachtsfall_Infografik_DINA3.html

8. Li Q, Guan X, Wu P et al (2020) Early transmission dynamics in Wuhan, China, of novel coronavirus-infected pneumonia. N Engl J Med. https://doi.org/10.1056/NEJMoa2001316

9. Jin YH, Cai L, Cheng ZS et al (2020) A rapid advice guideline for the diagnosis and treatment of 2019 novel coronavirus (2019nCoV) infected pneumonia (standard version). Mil Med Res $7(1): 4$

10. Poutanen SM, Low DE, Henry B et al (2003) Identification of severe acute respiratory syndrome in Canada. $\mathrm{N}$ Engl $\mathbf{J}$ Med 348(20):1995-2005

11. Huang C, Wang Y, Li X et al (2020) Clinical features of patients infected with 2019 novel coronavirus in Wuhan. China Lancet 395(10223):497-506

12. Chen Y, Chen L, Deng O (2020) The presence of SARS-CoV-2 RNA in the feces of COVID-19 patients. J Med Virol. https://doi. org/10.1002/jmv. 25825

13. Zhang W, Du RH, Liet B et al (2020) Molecular and serological investigation of 2019-nCoV infected patients: implication of multiple shedding routes. Emerg Microbes Infect 9(1):386-389

14. Homepage of Robert-Koch-Institute, Germany (2021) Number of registered SARS-CoV2-infections. Daily update. https://www.rki. de/DE/Content/InfAZ/N/Neuartiges_Coronavirus/Fallzahlen.html
15. Long QX, Liu BZ, Deng HJ et al (2020) Antibody responses to SARS-CoV-2 in patients with COVID-19. Nat Med 26(6):845848. https://doi.org/10.1038/s41591-020-0897-1. (Epub 29 Apr 2020)

16. Homepage of German Association of General Medicine (DEGAM) (2021) https://webcache.googleusercontent.com/search?q=cache: p-Lk3bdcv8oJ:https://www.degam.de/files/Inhalte/Degam-Inhalte/ Aktuelles/2020/Benefits/Coronavirus_MFA-Fragebogen.pdf+\& $\mathrm{cd}=9 \& \mathrm{hl}=\mathrm{de} \& \mathrm{ct}=\mathrm{clnk} \& \mathrm{gl}=$ de $\&$ client $=$ firefox $-\mathrm{b}-\mathrm{d}$

17. Huang X, Wie F, Hu L et al (2020) Epidemiology and Clinical Characteristics of COVID-19. Arch Iran Med. 23(4):268-271. Published 1 Apr 2020. https://doi.org/10.34172/aim.2020.09

18. Cervia C, Nilsson J, Zurbuchen Y et al. Systemic and mucosal antibody secretion specific to SARS-CoV-2 during mild versus severe COVID-19. https://doi.org/10.1101/2020.05.21.108308

19. Zhonghua Liu Xing Bing Xue Za Zhi. The epidemiological characteristics of an outbreak of 2019 novel coronavirus diseases (COVID-19) in China. 41:145-151. https://doi.org/10.3760/ cma.j.issn.0254-6450.2020.02.003

20. Shrock E, Fujimura E, Kula T (2020) Viral epitope profiling of COVID-19 patients reveals cross-reactivity and correlates of severity. Science. 27;370(6520):eabd4250. https://doi. org/10.1126/science.abd4250. Epub 29 Sep 2020

21. Guo Y, Cao QD, Hong ZS et al (2020) The origin, transmission and clinical therapies on coronavirus disease 2019 (COVID-19) outbreak - an update on the status. Mil Med Res 7:11. https://doi. org/10.1186/s40779-020-00240-0

22. Chan JF, Yuan S, Kok KH et al (2020) A familial cluster of pneumonia associated with the 2019 novel coronavirus indicating person-to-person transmission: a study of a family cluster. Lancet S0140-6736(20):30154-30159. https://doi.org/10.1016/ S0140-6736(20)30154-9

23. Yasuhara J, Kuno T, Takagi $\mathrm{H}$ et al (2020) Clinical characteristics of COVID-19 in children: a systematic review. Pediatr Pulmonol 55(10):2565-2575. https://doi.org/10.1002/ppul.24991 (Epub 4 Aug 2020)

24. Balasubramanian S, Rao NM, Goenka A et al (2020) Coronavirus disease 2019 (COVID-19) in children - what we know so far and what we do not. Indian Pediatr 57(5):435-442. https://doi. org/10.1007/s13312-020-1819-5 (Epub 9 Apr 2020)

25. Kohmer N, Rabenau H, Ciesek S (2020) SARS-CoV-2: Der richtige Nachweis. Dtsch Arztebl. 117(17): A-866 / B-729. https:// www.aerzteblatt.de/archiv/213661

26. Zhong L, Chuan J, Gong B et al (2020) Detection of serum IgM and IgG for COVID-19 diagnosis. Sci China Life Sci 63:777-780. https://doi.org/10.1007/s11427-020-1688-9

27. Li Z, Yi Y, Luo X et al (2020) Development and clinical application of a rapid IgM-IgG combined antibody test for SARSCoV-2 infection diagnosis. J Med Virol. https://doi.org/10.1002/ jmv. 25727

28. Zhao J, Yuan Q, Wanget $\mathrm{H}$ et al (2019) Antibody responses to SARS-CoV-2 in patients of novel coronavirus disease. Clin Infect Dis. 2020 Mar 28; ciaa344. https://doi.org/10.1093/cid/ciaa344

29. Li C, Ren L (2020) Recent progress on the diagnosis of 2019 Novel Coronavirus. Transbound Emerg Dis 67(4):1485-1491. https://doi.org/10.1111/tbed.13620 (Epub 2020 May)

30. Khan S, Nakajima R, Jain A et al. Analysis of Serologic CrossReactivity Between Common Human Coronaviruses and SARSCoV-2 Using Coronavirus Antigen Microarray. https://doi. org/10.1101/2020.03.24.006544

31. Liu L, Liu W, Wang S, Zheng S. A preliminary study on serological assay for severe acute respiratory syndrome coronavirus 2 (SARS-CoV-2) in 238 admitted hospital patients. Microbes Infect. https://doi.org/10.1016/j.micinf.2020.05.008 